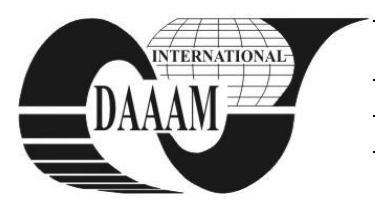

Annals of DAAAM for 2012 \& Proceedings of the 23rd International DAAAM Symposium, Volume 23, No.1, ISSN 2304-1382 ISBN 978-3-901509-91-9, CDROM version, Ed. B. Katalinic, Published by DAAAM International, Vienna, Austria, EU, 2012 Make Harmony between Technology and Nature, and Your Mind will Fly Free as a Bird Annals \& Proceedings of DAAAM International 2012

\title{
NANOMATERIALS IN SURFACE TREATMENT OF THE WOOD
}

\author{
HORMAN, I[zet]; SAPCANIN, A[ida]; AZEMOVIC, E[sed] \& PAZALJA, M[irha]
}

\begin{abstract}
According to the current development dynamics of nanostructured-materials area one can conclude that all the advantages of these materials will be economically exploited in the near future. This refers to the replacement of current materials and improvement of their properties, but also to the use of their completely new properties. This paper presents the research results of decorative protective films formed from nanostructured materials. Standard methods for testing the decorative protective films were implemented: hardness, resistance to mechanical damage, adhesion and water permeability. Test results have shown that nanomaterials intended for surface treatment of products for outdoor use show increased resistance to mechanical damage and water absorption, as well as satisfactory hardness and adhesion.

Keywords: nanomaterials, surface treatment, film, wood
\end{abstract}

\section{INTRODUCTION}

Traditional method of protection of wooden surfaces from deterioration is applying various types of materials that form a solid film while hardening. Coating on the outside of wooden building elements makes the interface between the wooden surface and the external environment, (Axelsen, S.T., 2008).

Since the wooden surface is subject to degradation due to the effects of various external factors, the thin film layer is expected that in addition to the aesthetic, also fulfills demanding protective function. The properties of wood such as dimensional stability, water resistance, resistance to biological degradation, resistance to ultraviolet and visible light, and even mechanical properties of wood can be improved by some of the modification procedures (Volinsky, A.A.,2002)

Lately, we can testify that terms nanomaterials and nanoparticles are occurring more frequently as terms for new materials for surface wood treatment. Nano layers are nanometer thin layers whose application is controlled or they organize themselves on the surface of the substrate, (Grigore, E., 2005). Namely, the solid particles of nanometer size can be dispersed in the lacquer and improve its properties, such as water resistance and additionally the resistance to ultraviolet radiation, and still remain invisible.

\section{NANOTECHNOLOGY}

Although the title of this chapter suggests science fiction, amounts to be invested in this area of technology are greater than the gross national product of many countries.

Nanotechnology deals with materials and processes of a billionth of a meter in size, on which the well-known materials can show very different behavior at the macroscopic level. Conceptually, nanosciences and nanotechnology refers to the sciences and technology that extends from about 100 nanometers to atomic order of magnitude $(\sim 0.2 \mathrm{~nm})$, and to the physical phenomena and properties of materials observed at work in this field of size. Nanomaterials are substances which contain extremely small particles, whose size ranges from 1 nanometer, that is $1 \cdot 0^{-10}$ meters. Substances containing nanoparticles often have a completely different, new properties, but nanotechnologies can significantly improve or repair the deficiencies of already existing materials, (Grigore, E., 2005).

Radical change in nanotechnology happens with the advent of scanning - tunneling microscope (scanning tunneling microscope) STM, Fig. 1.

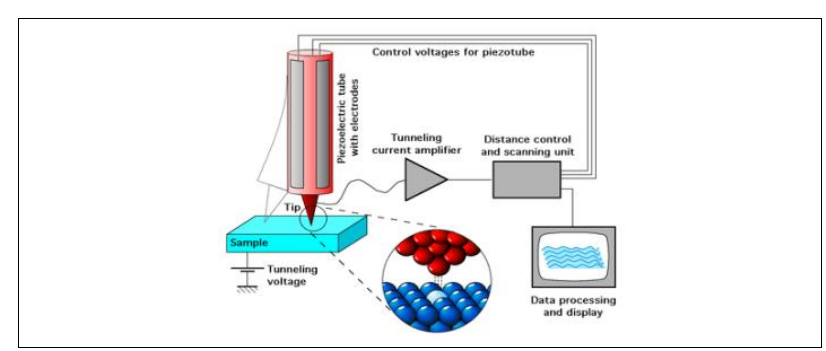

Fig. 1. Idealized image of the STM tip above the surface

STM is a device to "see" the surfaces of conductive materials with atomic precision and resolution of 0.2 nanometers. The basic element of the STM is the sharp tip made of the tungsten, platinum, iridium or some other conductive material. The top is (mostly) specifically engineered wire that is sharpened with special chemical or mechanical treatments, so that in ideall situation the top of the wire consists of just one or a few atoms. The tip is controlled by a computer which allows its precise adjustment with regard to the surface to be observed by STM.

Electric voltage is applied between the tip and the material, resulting in a small current when the tip is close to the surface, but only in the case when the material under investigation is visible. As the tip moves across the surface (in the $x-y$ plane), the current between the tip and the material is changed. Change in the current as a function of $(\mathrm{x}, \mathrm{y})$ position of the peak can be interpreted as a "picture" of the surface.

Atomic force microscope (AFM) Fig. 2., is another discovery that contributes to the development of nanotechnology. The working principle of AFM is: tiny probe (thread or tip pyramidal shape with a width from 2 to $30 \mathrm{~nm}$ ) is supplied to the direct contact with the 
sample. After that, it moves towards the end of the lever, which is bent as the tip moves along the relief surface of the sample. The shift in the vertical direction is measured by reflection of laser beam from the top of the lever.

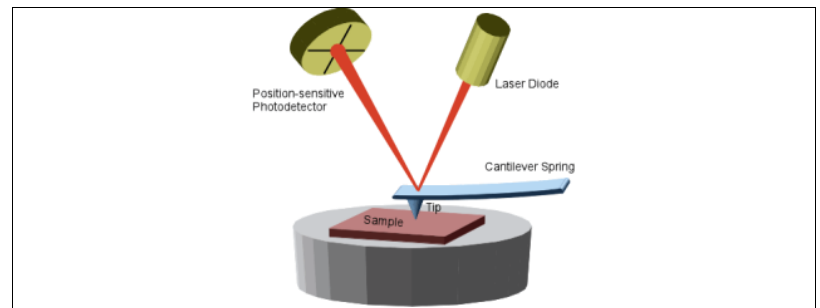

Fig. 2. Idealized image of AFM process

Unlike the STM, AFM does not measure current peaks between the microscope and the sample, but the force that acts between them. AFM provides a threedimensional surface profile. Along with electron microscopy, AFM and STM are the major devices for research and construction of nanostructures.

\section{MATERIALS AND METHODS}

In recent years, there have been great changes when it comes to production and use of products for wood surface treatment. An increasing attention is paid to systems that pollute the environment less, require less energy and produce quality films.

For the experimental analysis of the main features of the decorative protective films, nanocoating based on water with the addition of nanoparticles (aluminum oxide) was used. Nanomaterial is applied on the surface by the method of pneumatic dispersion and it forms liquid coating on the surface. Ground on which the film is applied is fir/spruce, processed with emery paper of 150 grit size. Among the investigated nanomaterials some basic properties are tested in liquid form: mass density, dry substance content, viscosity and film thickness in liquid form with room temperature of $210 \mathrm{C}$ and relative air humidity $51 \%$. The mass density implies mass per unit volume of a material. With liquid material surface treatment mass density is expressed as $\mathrm{g} / \mathrm{cm} 3$ and is called the mass density or density. The density of liquid materials is determined by using the pycnometer, Fig. 3. It is a container of standard density and weight of its own.

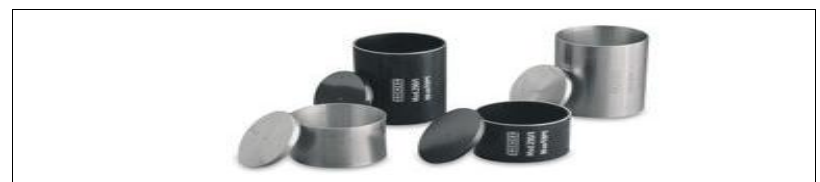

Fig. 3. Appearance of pycnometer for measuring the density of the liquid material (1) :

The mass density is calculated according to equation

$$
\rho=\frac{m_{1}-m}{v}
$$

Where:

$\rho$ - density of material $\left[\frac{\mathrm{g}}{\mathrm{m}^{2}}\right]$,

$\mathrm{m}_{1}$ - mass of pycnometer in [g],

$\mathrm{m}$ - mass of empty pycnometer in [g],

$\mathrm{V}$ - volume pycnometer in $\left[\mathrm{cm}^{3}\right]$.
Under the dry matter content we mean the totality of all the material in a liquid material that remains after the completion of the cure process - drying. These are materials that form a hard cover - film. The mass of material for testing, drying temperature and duration are dependent on the type and hemizm of liquid material.

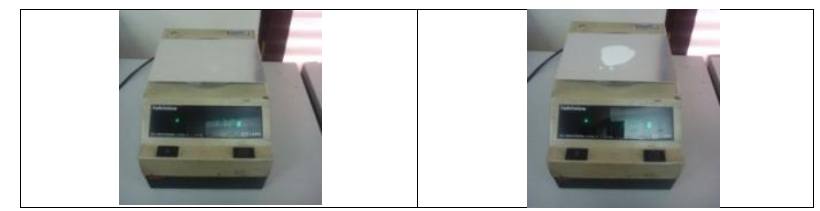

Fig. 4. Measuring the weights of the tube with and without polish

It is usually taken $2-5 \mathrm{~g}$ of material, drying is usually at $105 \pm 2{ }^{\circ} \mathrm{C}$, and the duration of drying to constant weight. Mass balance has been taken to a glass plate whose weight was known in advance, Fig. 4 . The content of dry substances, that is, the content of volatile components is calculated according to formula (2) and (3) :

$$
\begin{aligned}
S_{m} & =\frac{m_{1}}{m_{2}} \cdot 100[\%] \\
S_{i} & =100-S_{m}
\end{aligned}
$$

Where:

$S_{m}$ - content of dry materials in [\%],

$S_{i}$ - content of volatile materials [\%],

$m_{1}$ - the weight of the dried materials [g],

$m_{2}$ - the weight of thebalanced liquid material in [g].

The essence of the method according to Brookfield is counter of tested material to reversing of spindle in the shape of cylinder or disc. Resistance to reversing of spindle is the function of the viscosity of the tested material, and it is read on a scale of the instrument. Turning of the spindle is done by electro motor with stepwise adjusting the number of turns: 0.5, 2.0, 4.0, 5.0, and $100.0 \mathrm{r} / \mathrm{min}$.

Wet film thickness is measured immediately after application of liquid material - polish. Manufacturers of materials for surface treatment do not recommend wet film thickness, and it is regularly recommended the amount of applying $\mathrm{g} / \mathrm{m} 2$. Measurement of film thickness and knowing the density of the applied material can be calculated the applying amount by expression (4):

$$
Q=\rho \cdot d\left[\frac{g}{m^{2}}\right]
$$

Where:

$\rho$ - density $\left[\frac{g}{m^{2}}\right]$,
$d$ - wet film thickness $[\mu m]$.

In this procedure was used meter with approx measuring range 0-1200 um, Fig. 5

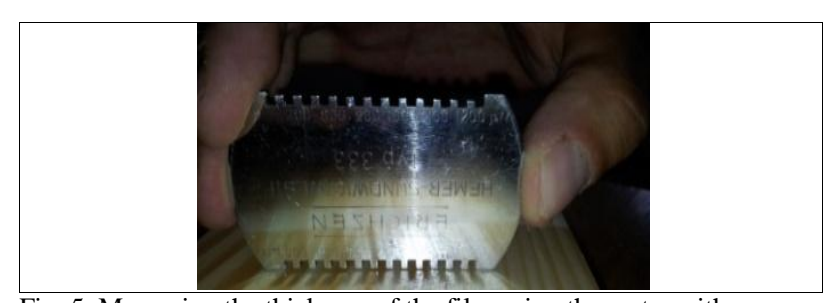

Fig. 5. Measuring the thickness of the film using the meter with approx 
The main goal and task of this work is verification of the basic features of decorative protective films formed from nanomaterials. For the film formed on the surface, the thickness, hardness, resistance to mechanical damage, adhesion and water permeability are analized.

Film thickness on the surface is a very important indicator of the quality of each surface treatment. Very thick or too thin films do not provide permanent protection and fast decay. Films should have optimum thickness, and it is different for different types of materials and forms of surface treatment. The method of measurement consists in removing (separation) of the narrow band of the film from the substrate and the direct measurement of film thickness on that site using comparators, as shown in Fig. 6.

Lack of mechanical methods, along with damage of the film and its difficult separation from the substrate, ordered from films with good adhesion.

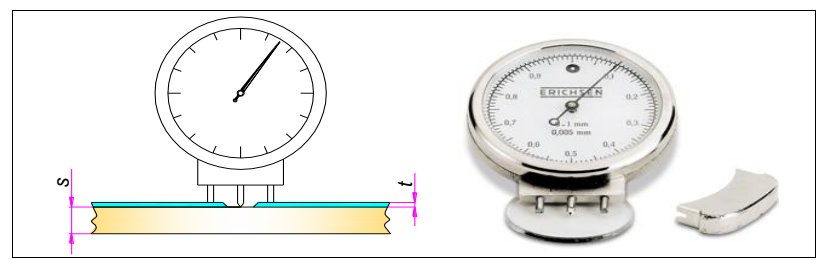

Fig. 6. Dry film thickness measurement

Comparator is calibrated before measurement on a special glass plate, ie. hand of comparator is brought to zero at the position when the needle tip of comparator in the same league with lugs. Testing and measuring dry film thickness was performed according to the standard EN ISO 2808.

By hardness in the physical sense we mean the resistance that the investigated body shows to penetrating into it by the second-hard body (Spitz, ball, roller, blades). Method according to Köning was used for measuring the coating hardness, Fig. 1. Measurement procedure was standardized according to BS 3900 E5.

The film is formed on a glass plate and placed on the instrument so that the two ball supports of the pendulum come on the film. The pendulum is moved from its equilibrium position and brought to the maximum left position. The pendulum is then released to swing freely, and the time of swaying is measured, it is $5^{\circ}-2^{\circ}$ (angular degrees).

Resistance to mechanical damage is determined by pulling a hard object of certain shape (scraper) under certain pressure over the tested material, and as a measure of the resistance, we take the force that makes the stump create a noticeable trace or scratch required by standard, $0.5[\mathrm{~mm}]$. The testing is regulated by the standard BS 3900, and the measurements were performed on the instrument according to Clemen's method, Fig. 8.

The specimen is set at the base and strengthened by the straps. The stump rests on the specimen, and the weight is set to a specific value on the scale. Then the device that leaves scratches on the film is turned on, and after finishing the scratching, the value of force is being increased by shifting weight to greater amount and then we scratch again. When we noticed that the stump leaves a trace on the specimen greater than $0.5(\mathrm{~mm})$, we abort the procedure and measure the width of the scratch trace .

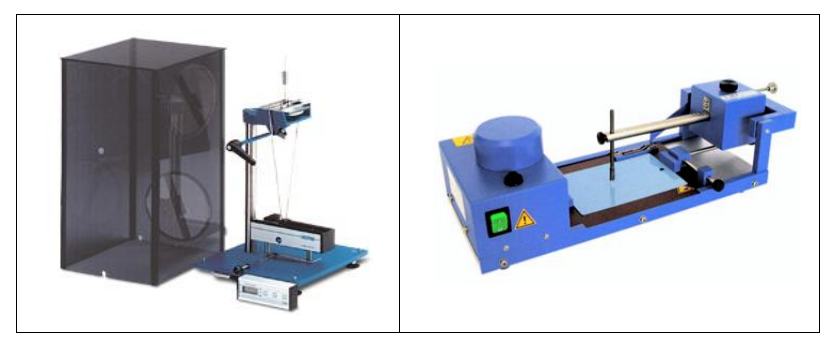

Fig. 7. Hardness testing according to Köning (left)

Fig. 8. Resistance testing according to Clemen(right)

The adhesion in surface treatment of wood implies property of forming strong link between the film and the substrate (wood). Adhesion is conditioned by forces of attraction of the film and substrate - wood molecules at the site of their mutual contact and depends on the physical - chemical properties of the film and substrate. The testing is based on cutting the film, which runs like lattice.

Lattice cutting is accepted by standard BS 3900 E6, and it is performed manually by a set of razor blades, Fig. 9. Razor blades are fixed at the same distance of 1 (mm). Cutting is performed in cross, so that a lattice of the cut film is formed with 25 squares.

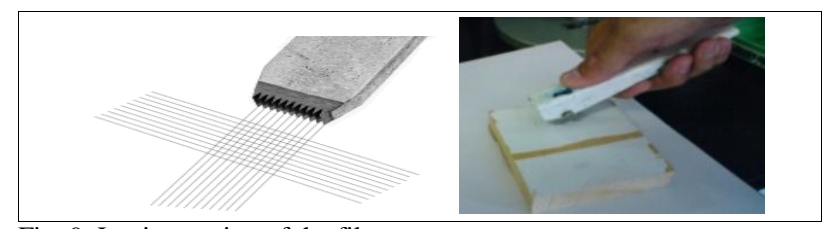

Fig. 9. Lattice cutting of the film

The permeability of water is resistance of the surface to water penetration. The tested nanomaterial is intended for outdoor use, so this ability is very important. Testing is done on pre-prepared probes with the dimension 20x20×20 (mm). Treated and untreated probes are submerged in water and in certain time intervals of 0.5 , $1,2,4,6,24$ and $48(\mathrm{~h})$, the amount of water absorption is controlled.

\section{RESULTS AND DISCUSSION}

Nanomaterials with the water-based nanoparticles (aluminum oxide) is tested in liquid and hardened state when the film is formed on the surface. Viscosity tests were carried out at $10-50 \%$ dilution, and the results are given in Fig. 10. Manufacturer of this nanomaterial provided in the data sheet the content of the dry substance within $42 \pm 1 \%$. Results for the five tested specimens are given in Fig. 11. Wet film thickness was measured for the three specimens with the result of 150 um. Based on the known wet film thickness and density

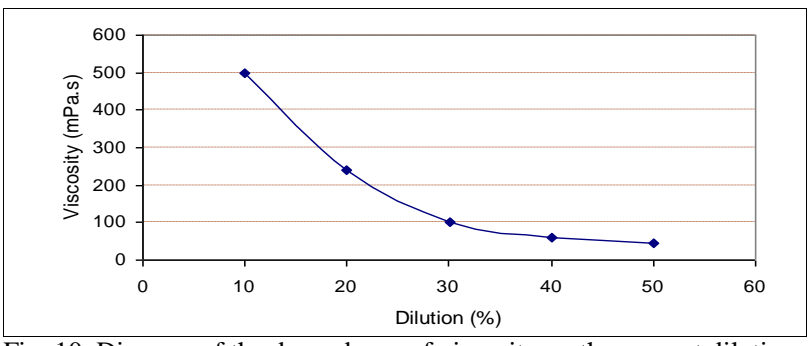

Fig. 10. Diagram of the dependence of viscosity on the percent dilution 


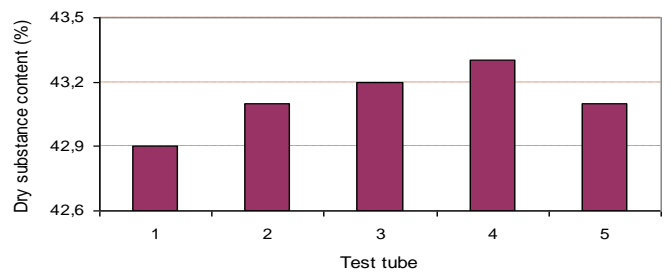

Fig. 11. The content of dry matter in the liquid form

of nanomaterials, application rate for the tested material is $156 \mathrm{~g} / \mathrm{m} 2$. Basic summary of results of nanomaterials in liquid form are given in Table 1.

\begin{tabular}{|l|c|}
\hline Property & \\
\hline Basic & Acrylic resin \\
\hline Specific gravity $\left[\mathrm{g} / \mathrm{cm}^{3}\right]$ & 1,04 \\
\hline Dry substance content [\%] & 43,1 \\
\hline Viscosity [mPa.s] & 500 \\
\hline
\end{tabular}

Tab. 1. Basic properties of nanomaterials in liquid form

Evaluation of the thickness of the film was done on three specimens with three layers, and measurements were made at three different locations at each layer. The results of these measurements are shown in the following Fig. 12.

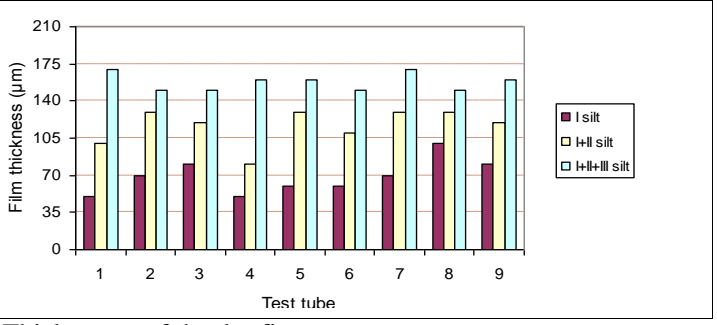

Fig. 12. Thicknesses of the dry firm

One of the properties of nanoparticles is to increase the hardness of films themselves. The hardness of the film after measuring on the pendulum is $t_{\mathrm{sr}}=35.4(\mathrm{~s})$ which is much more in relation to some standard lacquers that are used to protect wood in outdoor conditions. Fig. 13. shows the results of the hardness of dry films with three specimens. The results of testing the films on the mechanical damage are shown in the following table 2 .

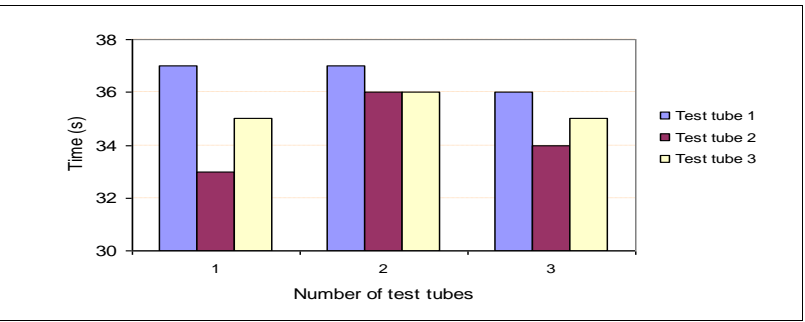

Fig. 13. Diagram of the hardness of dry films

\begin{tabular}{|c|c|c|}
\hline $\begin{array}{c}\text { The ordinal } \\
\text { number }\end{array}$ & $\begin{array}{c}\text { Traces visible in } \\
\text { clear sight Force }(\mathrm{N})\end{array}$ & $\begin{array}{c}\text { Track of } 0.5(\mathrm{~mm}) \\
\text { Force }(\mathrm{N})\end{array}$ \\
\hline 1. & 2 & 8 \\
\hline 2. & 2 & 8 \\
\hline
\end{tabular}

Tab.2. Film resistance to mechanical damage

As we can see from the results obtained in the table, nanomaterials' resistance to mechanical damage is extremely high and the first visible traces of scratching occur at a load of $2 \mathrm{~N}$, while the track of $0.5 \mathrm{~mm}$ width was measured only when the force of $8 \mathrm{~N}$ was applied. Adhesion testing is according to standard BS 3900 E6, which prescribes four classes of quality (A, B, C, and D). Therefore, the investigated samples belong to class $\mathrm{B}$ because the edges of the incision were partially truncated and not even one square of the film was lost.

For comparison purpose, surface-treated and untreated surfaces were tested on water permeability. Test results are given in Fig. 14. Water absorption of untreated probes during the first $30 \mathrm{~min}$ was about $1.1 \mathrm{~g}$, while with the treated probes water absorption was about $0.6 \mathrm{~g}$, which is about $50 \%$ less than in raw probes.

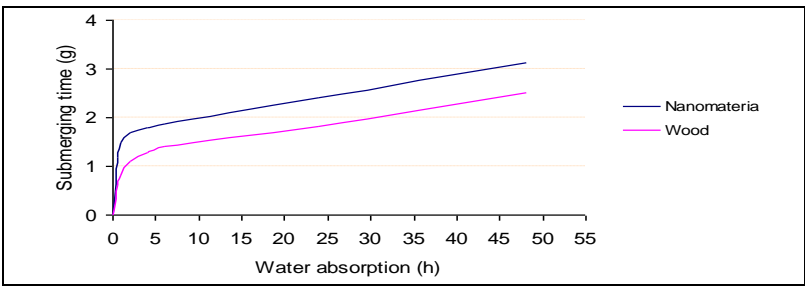

Fig. 14. Diagram of the water permeability of treated and untreated probes up to $48(\mathrm{~h})$

It can be concluded from Fig. 5 that the water absorption of processed specimens during the entire time interval was reduced in comparison to untreated specimens, classifying these lacquers with nanoparticles as materials suitable for the protection of wood exposed to external influences.

\section{CONCLUSION}

Analyzing the data obtained under these tests we come up with the following important conclusions:

- Nanomaterials which are used in surface protection of wood exposed to external influences increase the resistance to water and moisture absorption.

- Resistance to mechanical damage such as surface hardness and adhesion are significantly increased by using nanomaterials.

- The task of the future examining of nano substances is its ability and use in the real technological processes.

- Ability to achieve desired properties according to the requirements of the application is the basis for the further development of nanotechnology production of nanostructured materials, which are ascribed a key role for the entire 21 st century.

\section{REFERENCES}

[1] Axelsen, S.T.; et al. (2008). Topcoat flaking - a mechanism study. Party 1; Laboratory testing internal stress, mechanical properties, adhesion and ageing, Corrosion, March16-20, New Orleans, USA, Number of pages 14

[2] Grigore, E.; Ruset, C.; Short,; Hoeft, D.; Dong, H.; Li, X.Y. and Bellt, T. (2005) In situ investigation of the internal stress within the nc-Ti2N/nc-TiN nanocomposite coatings produced by a combined magnetron sputtering and ion implantation method, Surface and Coatings Technology, Vol. 200, Iss. 1-4, Oct 2005, pp. 744-747, ISSN O257-8972

[3] Vela, J.B.; Adhihetty, I.S.; Junker, K.; and Volinsky, A.A. (2003) Mechanical Propertis and Fracture Toughness of Organe - silicate Glass (OSG) Low-k Dielectric Thim Films for Microelectronic Application, Int. Journal of Fracture, Vol 119, No.4, pp 487- 499

[4] Volinsky, A.A.; Moody, N.R.; Gerberich, W.W. (2002) Interfacial Toughness Measuremens for Thin Films on Substrates, Act Mater., Vol. 50/30, pp. 441-466 